\title{
TRANSLUMINAL ENDOVASCULAR BRANCHED GRAFT PLACEMENT FOR A PSEUDOANEURYSM: RECONSTRUCTION OF THE DESCENDING THORACIC AORTA INCLUDING THE CELIAC AXIS
}

Kanji Inoue, MD, ${ }^{\mathrm{a}}$ Tomoyuki Iwase, MD, ${ }^{\mathrm{b}}$ Mitsuru Sato, $\mathrm{ME},{ }^{\mathrm{c}}$ Yuki Yoshida, MT, ${ }^{\mathrm{c}}$ Katsuya Ueno, RT, Shunich Tamaki, MD, ${ }^{\mathrm{b}}$ and Ario Yamazato, MD, ${ }^{\mathrm{a}}$ Kyoto, Japan

$\mathrm{We}^{1}$ have previously reported a successful transluminal endovascular placement of a branched graft that had a sidearm extending into the left subclavian artery for repair of type B aortic dissection. The sidearm was properly positioned by catching and pulling back the free end of a detachable wire attached to its end by means of a gooseneck snare wire, which was percutaneously inserted through the left brachial artery. However, it is impossible to place sidearm grafts into the celiac axis and renal arteries in a similar manner. In this article, we describe a new method of inserting a sidearm into the celiac axis and report successful transluminal endovascular repair of a pseudoaneurysm with the use of the branched graft.

A 73-year-old man with severe chronic renal failure requiring hemodialysis had previously undergone surgical repair of an aneurysm of the descending thoracic aorta. However, a pseudoaneurysm of the descending thoracic aorta resulting from dehiscence of the suture line at the proximal and distal anastomoses after composite graft surgery had continued to dilate, reaching $80 \mathrm{~mm}$ in diameter. Although surgical treatment was attempted, the effort was given up because of marked adhesions caused by a previous thoracotomy. He was admitted to our hospital for endovascular treatment of the pseudoaneurysm.

The structure of the Inoue endovascular graft was previously described in detail. ${ }^{2}$ The graft was constructed from a Dacron polyester fabric cylinder and the surface was supported by multiple rings of extra-flexible nickel titanium wire. The patient gave informed consent in conformance with the protocols approved by the institutional review board of Takeda Hospital.

Endovascular grafting with the straight graft was performed on June 28, 1995. Although the proximal communication was completely obliterated after the procedure, the distal communication persisted because the distal orifice of the pseudoaneurysm was in close proximity to the celiac axis. Although transluminal embolization was

From the Departments of Cardiovascular Surgery ${ }^{\mathrm{a}}$ and Cardiology, ${ }^{\mathrm{b}}$ the Clinical Laboratory ${ }^{\mathrm{c}}$ and the Department of Radiology, ${ }^{\mathrm{d}}$ Takeda Hospital, Kyoto, Japan.

Received for publication Feb. 18, 1997; accepted for publication April 1, 1997.

Address for reprints: Kanji Inoue, MD, Department of Cardiovascular Surgery, Takeda Hospital, Higashiiru, Nishinotoin, Shiokojidori, Shimogyoku, Kyoto 600, Japan.

J Thorac Cardiovasc Surg 1997;114:859-61

Copyright $(1) 1997$ by Mosby-Year Book, Inc.

$0022-5223 / 97 \$ 5.00+0 \quad \mathbf{1 2} / \mathbf{5 4} / \mathbf{8 2 3 5 1}$ subsequently attempted to obliterate the residual distal communication and thereby the leakage reduced, the aneurysm was only partially thrombosed because of the patient's coagulopathy. Therefore, extending the graft into the celiac axis was necessary. A second endovascular grafting with a branched prosthesis was performed on June 6,1996 . The size of the tapered main graft was 24 $\mathrm{mm}$ in diameter at the proximal end, $27 \mathrm{~mm}$ at the distal end, and $50 \mathrm{~mm}$ in length. The sidearm was $8.5 \mathrm{~mm}$ in diameter and $15 \mathrm{~mm}$ in length.

A $22 \mathrm{~F}$ sheath was advanced to the descending thoracic

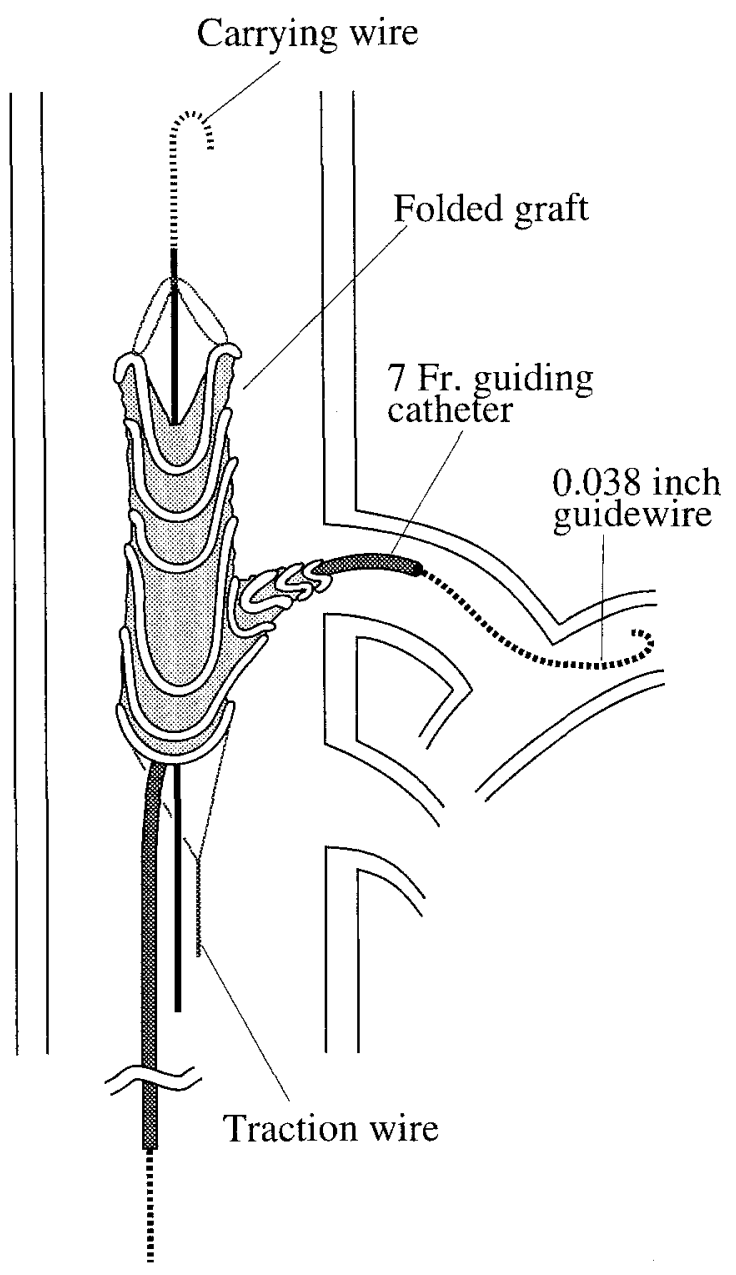

Fig. 1. The way in which the sidearm of the branched graft is placed into the celiac axis. 

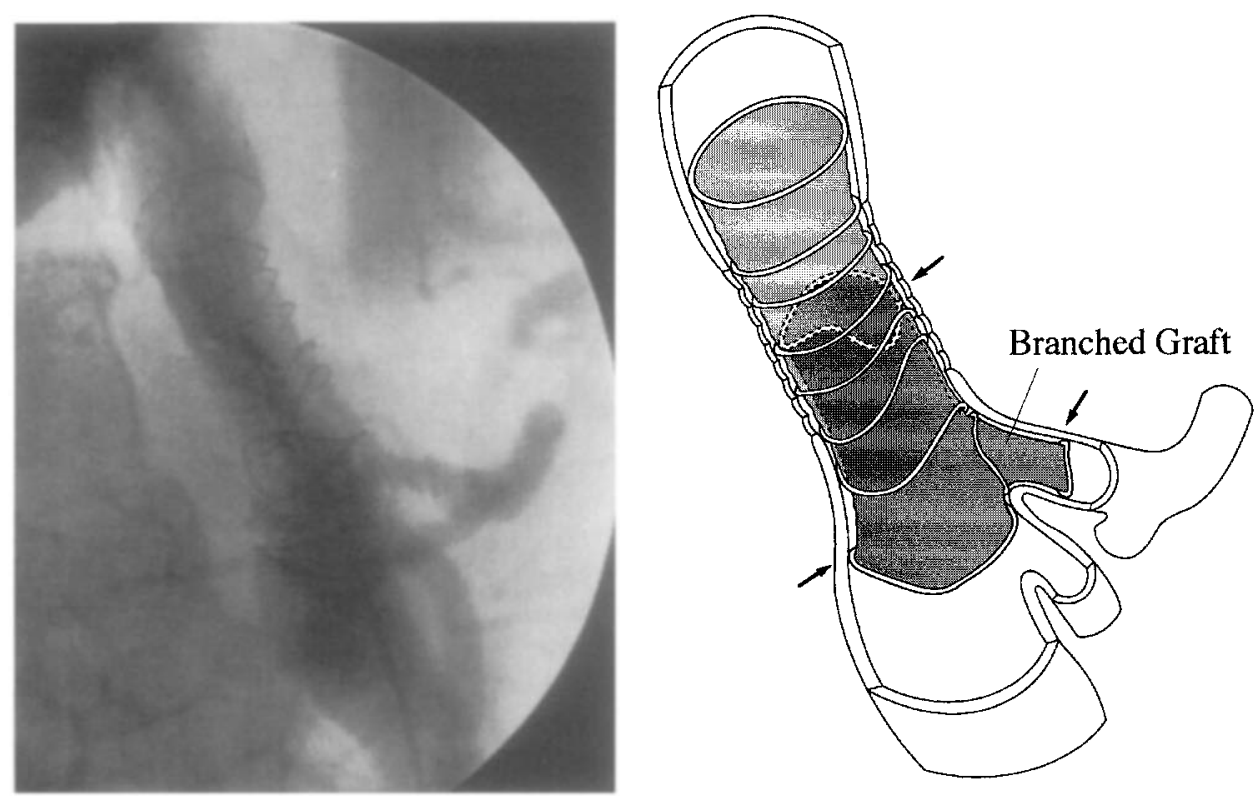

Fig. 2. Aortogram showing exclusion of a pseudoaneurysm. The upper end of the branched graft is placed into the earlier placed endovascular graft.

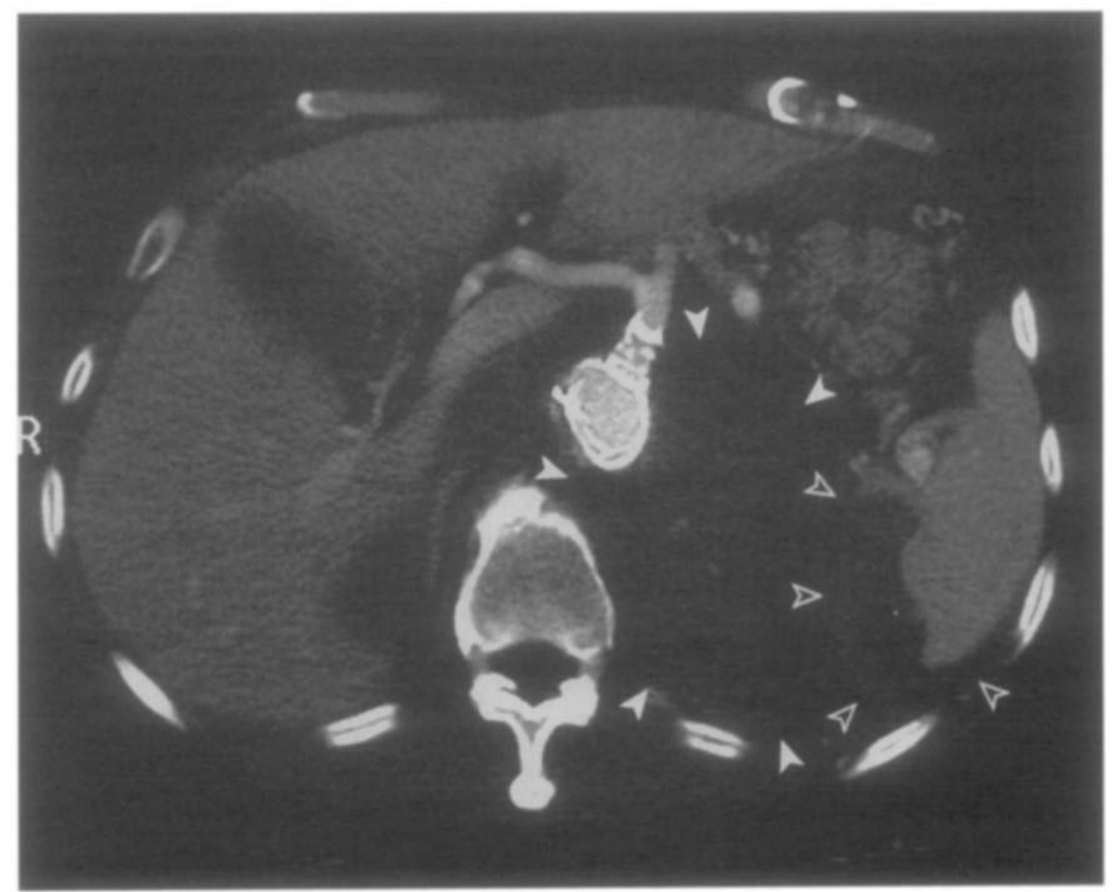

Fig. 3. Transaxial view of helical computed tomographic angiogram 1 week after the procedure shows a thrombosed pseudoaneurysm (solid arrowheads) and a perisplenic hematoma (open arrowheads).

aorta through the left femoral artery, which had been exposed under local anesthesia. Fig. 1 shows the method of placing the branched graft. The main graft and sidearm were individually folded using loops of thread and a nickel titanium wire in such a way that the graft should not spontaneously expand after its release from the sheath. A
$7 \mathrm{~F}$ detachable guiding catheter was inserted through the main graft into the sidearm and connected to the end of the sidearm. With the aid of carrying and traction wires, the folded branched graft was advanced close to the orifice of the celiac axis through the sheath. Then only the sheath was pulled back while the graft was held firm. ${ }^{2} \mathrm{~A}$ 
0.038 -inch guidewire was inserted into the guiding catheter and advanced deeply through the celiac axis into the splenic artery. The sidearm was introduced into the celiac axis over the guidewire by manipulating the guiding catheter. After the branched graft was in position, only the main graft was deployed by removal of the nickel titanium wire and pressed against the aortic wall by balloon inflation. The folded sidearm was then deployed in the same way. The guiding catheter was replaced with a balloon catheter and the sidearm was dilated completely. After the procedure, aortography showed the complete exclusion of the pseudoaneurysm and good flow of contrast medium through the branched graft (Fig. 2). Computed tomography, which was performed 1 week after the procedure, showed the thrombosed pseudoaneurysm and a perisplenic hematoma without bleeding (Fig. 3). The hematoma spontaneously resolved. No evidence of paraplegia, aneurysm enlargement, or significant graft stenosis had arisen during a follow-up period of 8 months. Follow-up computed tomography has demonstrated persistent exclusion of the pseudoaneurysm.

In recent years, the feasibility and effectiveness of endovascular grafting with straight and bifurcated grafts for the repair of aortic aneurysms have been reported..$^{3-5}$ We attempted reconstruction of the descending thoracic aorta including the celiac axis with a branched graft. The only complication was an injury of the splenic artery resulting from guidewire insertion. We believe this complication can be prevented by gently advancing a J-tipped guidewire into the arterial branches. Endovascular grafting with the use of branched grafts permits repair of true aneurysms and aortic dissections, including the origin of the major aortic arterial branches such as the renal arteries and celiac axis.

In the near future, endovascular grafting will become a life-saving treatment for patients, like our patient, who cannot undergo surgery. Although our short-term result has been favorable, a long-term evaluation on large numbers of patients will be necessary to prove the longterm effectiveness and safety of transluminal endovascular graft placement.

\section{REFERENCES}

1. Inoue $K$, Sato $M$, Iwase $T$, Yoshida $Y$, Tanaka T, Tamaki S, et al. Clinical endovascular placement of branched graft for type B aortic dissection. J Thorac Cardiovasc Surg 1996;112:1111-3.

2. Inoue $\mathrm{K}$, Iwase T, Sato M, Yoshida Y, Tanaka T, Kubota Y, et al. Clinical application of transluminal endovascular graft placement for aortic aneurysms. Ann Thorac Surg 1997;63: 522-8.

3. Parodi JC, Palmaz JC, Barone HD. Transfemoral intraluminal graft implantation for abdominal aortic aneurysms. Ann Vasc Surg 1991;5:491-9.

4. Dake MD, Miller DC, Semba CP, Mitchell RS, Walker PJ, Liddell RP. Transluminal placement of endovascular stentgrafts for the treatment of descending thoracic aortic aneurysms. N Engl J Med 1994;331;1729-34.

5. Chuter TAM, Green RM, Ouriel K, Fiore WM, DeWeese JA. Transfemoral endovascular aortic graft placement. J Vasc Surg 1993;18:185-97.

\section{SURGICAL EXCISION OF THE SINUS NODE IN A PATIENT WITH INAPPROPRIATE SINUS TACHYCARDIA}

Bahman Esmailzadeh, MD, ${ }^{a}$ Robert Bernat, MD, ${ }^{\mathrm{b}}$ Kai Winkler, MD, ${ }^{\mathrm{a}}$ Monika Meybehm, MD, ${ }^{\mathrm{c}}$ Dietrich Pfeiffer, MD, ${ }^{\mathrm{b}}$ and Paul Gerhard Kirchhoff, MD, ${ }^{a}$ Bonn, Germany

Inappropriate sinus tachycardia is a rare arrhythmia occurring in otherwise healthy persons, possibly owing to increased automaticity of the sinus node or an automatic

From the Departments of Cardiac and Vascular Surgery, ${ }^{\mathrm{a}} \mathrm{Car}-$ diology, ${ }^{\mathbf{b}}$ and Pathology, ${ }^{\mathrm{c}}$ University of Bonn Medical School, Bonn, Germany.

Received for publication March 3, 1997; accepted for publication May 22, 1997.

Address for reprints: Bahman Esmailzadeh, MD, Universitätsklinik und Poliklinik für Herz-und Gefäßchirurgie, Sigmund-Freud-Str. 25, 53105 Bonn-Venusberg, Germany.

J Thorac Cardiovasc Surg 1997;114:861-4

Copyright $\odot 1997$ by Mosby-Year Book, Inc.

$0022-5223 / 97 \$ 5.00+0 \quad \mathbf{1 2 / 5 4 / 8 3 4 1 1}$ atrial focus located near the sinus node. The abnormality also may be related to a defect in autonomic control of sinoatrial automaticity. ${ }^{1}$ The current report supports the findings of previous studies concerning the role of surgical treatment of patients with this condition. ${ }^{2}$

Clinical summary. The patient, a 36-year-old woman, had had persistent tachycardia for 4 years. She had previously undergone two unsuccessful catheter ablation attempts for her tachycardia in a highly experienced ablation center. First radiofrequency energy and then direct current were used. The ablations were followed only by a transient and moderate deceleration of the heart rate. She continued to have an accelerated pulse rate at rest, rarely below 100 beats/min. Even with mild exertion ( 25 watts) the heart rate exceeded 150 beats/min and the associated symptoms of chest tightness and dyspnea re- 